\title{
Velocity-Selective Arterial Spin Labeling
}

\author{
Eric C. Wong, ${ }^{1,2^{*}}$ Matthew Cronin, ${ }^{1}$ Wen-Chau Wu, ${ }^{1}$ Ben Inglis, ${ }^{3}$ Lawrence R. Frank, ${ }^{1}$ \\ and Thomas T. Liu ${ }^{1}$
}

In pathologies in which slow or collateral flow conditions may exist, conventional arterial spin labeling (ASL) methods that apply magnetic tags based on the location of arterial spins may not provide robust measures of cerebral blood flow (CBF), as the transit delay for the delivery of blood to target tissues may far exceed the relaxation time of the tag. Here we describe current methods for ASL with velocity-selective (VS) tags (termed VSASL) that do not require spatial selectivity and can thus provide quantitative measures of CBF under slow and collateral flow conditions. The implementation of a robust multislice VSASL technique is described in detail, and data obtained with this technique are compared with those obtained with conventional pulsed ASL (PASL). The technical considerations described here include the design of VS pulses, background suppression, anisotropy with respect to velocity-encoding directions, and CBF quantitation issues. Magn Reson Med 55:1334-1341, 2006. ㄷ 2006 Wiley-Liss, Inc.

Key words: cerebral blood flow; arterial spin labeling; velocity selective; transit delay; background suppression

In conventional arterial spin labeling (ASL) techniques, including both pulsed ASL (PASL) (1-5) and continuous ASL (CASL) (6-8), arterial blood is tagged by magnetic inversion or saturation proximal to the region of interest (ROI). Tagged blood then flows into the ROI, and the inflow is detected as a modulation of the longitudinal magnetization. In these techniques there is necessarily a spatial gap between the tagging location and the ROI. This gap results in a transit delay ( $\delta \mathrm{t}$ ) for the delivery of tagged blood to the ROI. The gap (and hence the delay) can be small for single-slice imaging, but is larger for multislice or volume acquisitions. The magnitude and variability of the transit delay in relation to the $T_{1}$ decay of the tag is one of the largest potential sources of errors in the quantitation of perfusion using ASL in the normal human brain $(5,6,9)$. In stroke and other pathologies in which flow may be slow or may follow circuitous collateral routes of delivery, $\delta$ t can be much larger than $T_{1}(10)$, which makes conventional ASL an impractical method for obtaining accurate measures of $\mathrm{CBF}$.

${ }^{1}$ Department of Radiology, University of California-San Diego, La Jolla, California, USA.

${ }^{2}$ Department of Psychiatry, University of California-San Diego, La Jolla, California, USA.

${ }^{3}$ Henry H. Wheeler, Jr., Brain Imaging Center, University of California-Berkeley, Berkeley, California, USA.

Grant sponsor: NIH; Grant number: R01 EB002096.

${ }^{\star}$ Correspondence to: Eric C. Wong, Ph.D., M.D., UCSD Center for Functional MRI, 9500 Gilman Drive, MC 0677, La Jolla, CA 92093-0677. E-mail: ecwong@ucsd.edu

Received 17 May 2005; revised 15 February 2006; accepted 15 February 2006

DOI 10.1002/mrm.20906

Published online 12 May 2006 in Wiley InterScience (www.interscience. wiley.com).

(c) 2006 Wiley-Liss, Inc.
We recently introduced a new ASL method in which the tag pulse is purely velocity-selective (VS) and not spatially-selective. This allows for the tagging of all flowing spins within a specified velocity range, regardless of location, and can in principle eliminate the problem of transit delays. We refer to this technique as VSASL, and in this work we describe the implementation of VSASL, as well as some of the considerations involved in the design of VSASL pulse sequences and the quantitation of perfusion using this technique. VSASL was introduced in abstract form in Ref. 11, and some of the issues addressed herein were described in Refs. 11-16. Norris and Schwartzbauer (17) suggested the use of VS pulses in ASL, but did not present an implementation of VSASL.

\section{THEORY}

In principle, the elements of a VSASL pulse sequence are similar to those of a conventional ASL experiment and include a tagging pulse that modifies the magnetization of inflowing arterial spins, followed by a delay (TI) to allow for inflow, and a rapid image acquisition. In the ideal case, a VS tag is applied that modulates the longitudinal magnetization of blood above a sharp cutoff velocity $V_{c}$. After a delay period TI during which the tagged blood flows down the arterial tree, an image (the tag image) is acquired of all spins whose velocities are below $V_{c}$. By imposing the restriction $V<V_{c}$ for the image acquisition by means of additional VS pulses, the resulting image includes tagged magnetization only from spins that decelerated during TI from above to below $V_{C}$. If the velocities in the arterial tree are monotonically decreasing, then the amount of tagged blood that appears in the image is simply TI - CBF, where $\mathrm{CBF}$ is the local cerebral blood flow (in the case of brain imaging). A second function of the velocity selectivity in the image acquisition is to act as a filter for arterial vs. venous blood. In general, venous blood accelerates, and while a large volume of venous blood with $V>V_{c}$ will be tagged, it will generally still be at $V>V_{C}$ at the time of image acquisition and will not be detected. Without velocity selectivity in the image acquisition, the VSASL signal would have a potentially large blood volume component, as opposed to being proportional to $\mathrm{CBF}$.

As in conventional ASL, the tag image samples the longitudinal magnetization of static tissue as well as the modulated magnetization of inflowing blood. This image can be alternated across TR periods with control images in which the VS tag was not applied, but the image acquisition is again restricted to $V<V_{c}$. Taking the difference between the tag and control images removes the static tissue component, and reflects only magnetization that was above $V_{c}$ at the time of the tag and below $V_{C}$ at the time of the image acquisition. This signal difference is the VSASL signal. 
FIG. 1. Left: Ideal velocity cutoff. Right: Imperfect velocity cutoff. In either case the total amount of tagged blood appearing in the image is $\mathrm{TI} \cdot \mathrm{CBF}$.
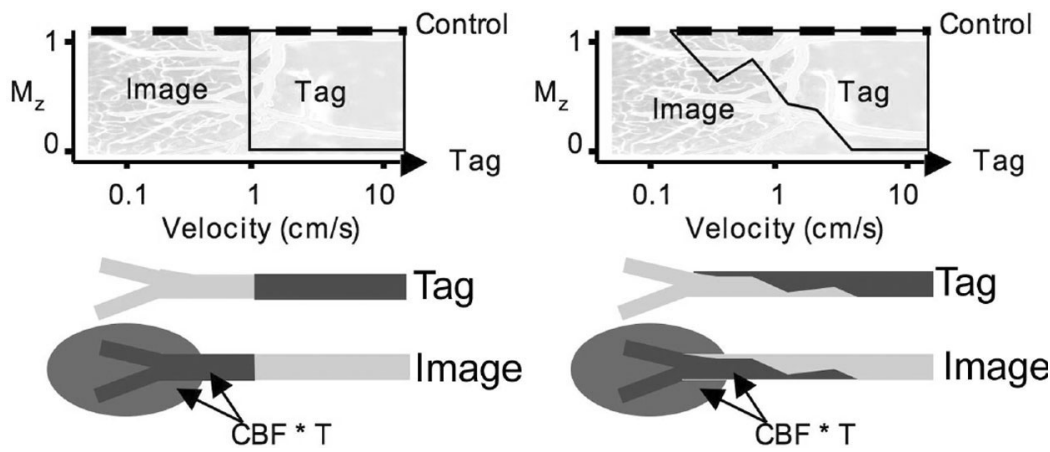

In the ideal case, the velocity selectivity is rectangular in profile, as shown on the left side of Fig. 1. If arterial blood decelerates monotonically, then the rate of flow of blood across the boundary defined by the velocity cutoff $V_{C}$ is equal to the local CBF, and the total blood delivered downstream will be TI - CBF (see Fig. 1). In practice, the transition from an upstream region that is fully tagged to a downstream area that is completely untagged will be more complex, both because the velocity selectivity is nonideal, and because any particular component of the flow may not be monotonically decelerating. This creates a transition zone in which blood is partially tagged during the tagging process and is partially excited during the imaging process (Fig. 1, right). However, as long as the spins in this transition zone clear the transition zone during the inflow delay TI, the total VSASL signal will still be proportional to TI - CBF. This is because the leading edge of the tag bolus is defined by the saturated portion of the tagging profile, while the trailing edge of the tag bolus is defined by the unsaturated portion of the same tagging profile. These imperfect edges are complementary and therefore define a bolus with total volume TI - CBF. Thus the total size of the bolus is relatively insensitive to the shape of the velocity-selectivity profile. However, this profile does determine how far up the vascular tree the tail end of the tag will appear in the VSASL image, and thus limits the spatial localization of the signal to the target tissue.

In order to maximize the VSASL signal, complete inversion of spins with $|V|>V_{C}$ would be desired, but since there are no pulse trains currently known to produce inversion across a wide range of velocities, the discussion here focuses on VS saturation. A simple sequence that provides VS modulation of the longitudinal magnetization is $90_{\mathrm{x}}-G-180_{\mathrm{y}}-G-90_{-\mathrm{x}}(11,13)$, which can be implemented using simple hard RF pulses. This spin-echo sequence produces a modulation of $M_{z}$ given by $M_{z}=M_{o} \cos (\beta v)$ where $\beta=\gamma G \delta \Delta \gamma$ is the gyromagnetic ratio, $G$ is the gradient amplitude, $\delta$ is the gradient duration, $\Delta$ is the separation between the leading edges of the gradient lobes, and $v$ is the velocity. In the presence of laminar flow, where the distribution of flow velocities is uniform from 0 to twice the mean velocity $v$, the modulation becomes $M_{z}=$ $M_{o} \operatorname{sinc}(2 \beta v)$. The first zero crossing of this function occurs at $V_{c}=\pi / 2 \beta$. This method has no spatial selectivity, is insensitive to resonance offset, and can be made short in time and low in SAR. However, in the simplest implementation using linear RF pulses, it is sensitive to $B_{1}$ inhomogeneity and can have significant diffusion weighting. At
$1.5 \mathrm{~T}$ in the brain, the $B_{1}$ homogeneity is typically sufficient to allow for the use of hard pulses in VSASL (18). At 3T, however, we have found that the typical $B_{1}$ variation across the brain creates subtraction errors between tag and control images that can be much larger than the VSASL signal when linear pulses are used, consistent with findings in Ref. 13. In Ref. 13 a phase cycling scheme was used to reduce these subtraction errors. This scheme can successfully reduce these errors, but results in reduced tagging efficiency and requires the use of nonzero velocity weighting in the control condition.

\section{MATERIALS AND METHODS}

\section{VS Tagging Pulse Train}

For VS tagging, a modified spin-echo pulse train was used that combines hard $90^{\circ}$ pulses with an adiabatic refocusing method, first introduced in Ref. 19, that uses a pair of hyperbolic secant (sech) inversion pulses to generate a spin echo. The composite refocusing pulse has very low sensitivity to resonance offset and $B_{1}$ inhomogeneity, and thus serves as a robust transparent pulse for stationary spins. The hard pulses at the beginning and end of the pulse train are linear with $B_{1}$, but because the double sech refocusing returns magnetization generated by the first hard pulse to the same position prior to the second hard pulse, the magnetization will be accurately returned to the $+\mathrm{Z}$ axis regardless of the initial flip angle. Thus, the tagging efficiency will be proportional to $\sin (\phi)$, where $\phi$ is the local flip angle of the hard pulses, but $B_{1}$ inhomogeneity will not generate subtraction errors in static tissue between tag and control images. The duration of this fourRF-pulse train can be significant compared to the $T_{2}$ of blood, and in order to reduce the time it is desirable to reduce the duration of the sech pulses. One can minimize the duration of a sech pulse without affecting adiabaticity by transforming it into a rectangular pulse by nonlinear scaling of the time axis with preservation of the pulse area (20). This results in a shorter pulse with a larger bandwidth than the original, but the frequency offset approaches infinity at the ends of the pulse. To moderate this effect and avoid potential problems with the infinitely rapid turn-on of the $\mathrm{RF}$ system, we transform the sech pulse into a pulse of the form $\cos ^{n}(\mathrm{t})$ on the interval- $\pi / 2<$ $\mathrm{t}<\pi / 2$, with $\mathrm{n}$ providing a tunable parameter that allows the pulse to approach rectangular as $n->0$. For the pulses used in this study, and shown in Fig. 2, $\mathrm{n}=0.2$. 


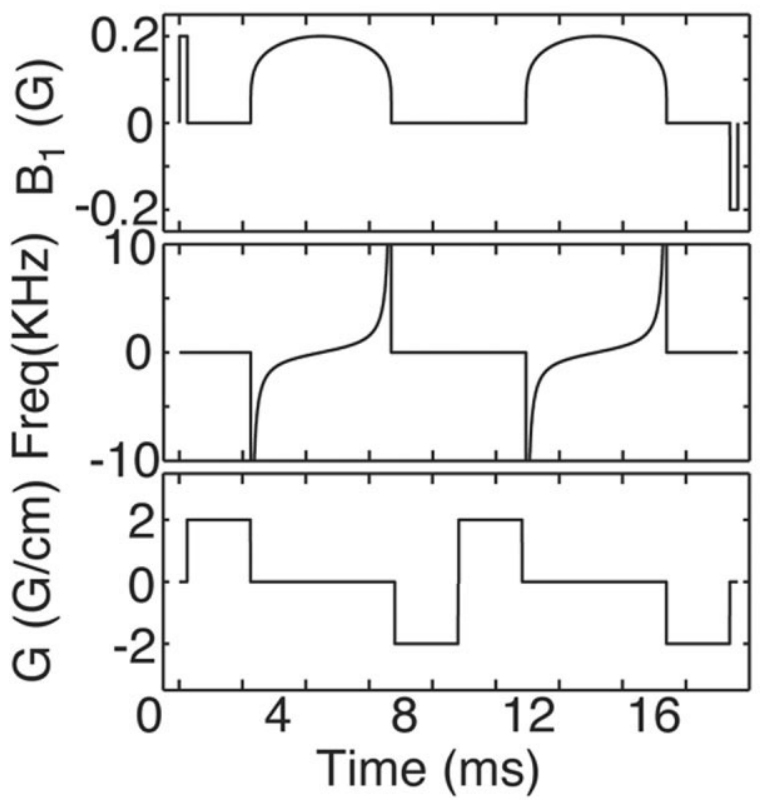

FIG. 2. Double sech VS pulse train with a flow-encoding scheme that uses four gradient pulses to reduce eddy-current effects.

We found in phantom studies that when we used the four-RF-pulse train described above, with gradient pulses in the first and third periods between RF pulses, at the lowest $V_{C}$ of $1 \mathrm{~cm} / \mathrm{s}$, significant subtraction errors between the tag and control images still existed that were not attributable to either diffusion weighting or $B_{1}$ inhomogeneity. This artifact may be related to gradient amplifier hysteresis (21), long-time constant eddy currents, or positivenegative gradient asymmetry, and was greatly reduced by using the four-gradient-pulse scheme described by Reese et al. (22) for reducing eddy-current effects in diffusion imaging. This four-pulse sequence is shown in Fig. 2.

In addition to the velocity-selectivity profile, the tagging pulse train is characterized by a tagging efficiency $\alpha$, which in this case is likely dominated by $T_{2}$ decay during the tagging pulse train. For this work we assumed $\alpha$ $=e^{-T E_{t a g} / T_{2}}$, where $\mathrm{TE}_{\mathrm{tag}}$ is the total duration of the tagging pulse train, and $T_{2}$ is that of arterial blood.

\section{Contamination of the VSASL Signal by CSF}

Under ideal circumstances the VSASL difference signal is dominated by the modulation of the signal from inflowing blood spins. However, both diffusion and motion unrelated to blood flow will also lead to differences between the tag and control VSASL signals. The diffusion sensitivity of the tag pulse train is characterized by the $b$-value of the sequence, while the sensitivity to coherent motion is characterized by $V_{c}$. In the brain, CSF will generally produce the largest subtraction errors from both diffusion and motion, as the diffusion coefficient and velocity of CSF flow are higher than those of other nonblood tissues.

For bipolar flow/diffusion-weighting gradients, $V_{C}$ and diffusion $b$-value have different dependences on $G, \delta$, and $\Delta$ (the amplitude, duration, and separation of the gradient lobes, respectively). The flow moment, which is propor- tional to $1 / V_{c}$, scales linearly with these three parameters, while the $b$-value scales with the square of $G$ and $\delta$, and linearly with $\Delta$. Thus for a fixed value of $V_{c}$, as $\Delta$ increases, the $b$-value decreases, and in order to reduce the diffusion weighting for a given value of $V_{c}, \Delta$ should not necessarily be minimized. The trade-off is that larger $\Delta$ results in greater $T_{2}$-related attenuation of the tagged magnetization. In the initial implementations of VSASL $(11,13)$, a hard pulse $90-G-180-G-90$ scheme was used for minimum $T_{2}$ weighting. This led to significant $b$-values and a concern about tag-control subtraction errors due to diffusion weighting, particularly in CSF. A property of the double sech pulse train that we are currently using is that the gradient separation is necessarily larger than the minimum separation using the $90-G-180-G-90$ scheme. While this is a disadvantage in terms of $T_{2}$ decay during the tagging process, it reduces the diffusion weighting significantly, and a double sech tagging pulse train of minimum duration generates a diffusion weighting that is likely negligible at our typical operation point of $V_{C}=2 \mathrm{~cm} / \mathrm{s}$. Table 1 shows calculated $b$-values and levels of diffusion attenuation for the minimum-time hard pulse and double sech tagging pulse trains as a function of $V_{c}$. The hard pulse train can of course be lengthened to provide reduced diffusion weighting at a given $V_{C}$ if this is desired.

\section{Background Suppression (BGS)}

Because the ASL signal is made up of relatively small differences between tag and control states, fluctuations in the much larger static tissue signal due to subject motion and physiologic pulsations can dominate the ASL signal. Suppression of the static tissue signal has been shown to be an effective method for improving signal stability in ASL (23-25). When BGS was used with VSASL, two adiabatic (sech) inversion pulses were placed $450 \mathrm{~ms}$ and $1550 \mathrm{~ms}$ prior to image acquisition to minimize the signal from tissues with $T_{1}$ in the range of 700-1600 ms. Because the tagging pulses in VSASL are spatially nonselective, nonselective inversion pulses were used for BGS.

Table 1

Diffusion Attenuation of Tag Pulse Train

\begin{tabular}{|c|c|c|c|c|c|}
\hline $\mathrm{V}_{\mathrm{c}}(\mathrm{cm} / \mathrm{s})$ & 1 & 2 & 4 & 8 & 16 \\
\hline \multicolumn{6}{|c|}{ Hard pulses min. duration } \\
\hline Gradient $(\mathrm{G} / \mathrm{cm})$ & 4.00 & 4.00 & 4.00 & 2.14 & 1.07 \\
\hline$\delta(\mathrm{ms})$ & 2.80 & 1.76 & 1.06 & 1.00 & 1.00 \\
\hline$\Delta(\mathrm{ms})$ & 5.20 & 4.16 & 3.46 & 3.40 & 3.40 \\
\hline $\begin{array}{l}\mathrm{b}\left(\mathrm{s} / \mathrm{mm}^{2}\right) \\
1-\mathrm{e}^{-\mathrm{bD}} \text { (brain) }\end{array}$ & 4.0 & 1.3 & 0.43 & 0.13 & 0.03 \\
\hline $\begin{array}{c}(\%) \\
1-e^{-b D}(C S F)\end{array}$ & 0.40 & 0.13 & 0.043 & 0.013 & 0.003 \\
\hline \multicolumn{6}{|l|}{ Double sech } \\
\hline Gradient (G/cm) & 2.20 & 1.64 & 0.82 & 0.41 & 0.21 \\
\hline$\delta(\mathrm{ms})$ & 1.40 & 1.00 & 1.00 & 1.00 & 1.00 \\
\hline$\Delta(\mathrm{ms})$ & 19.0 & 17.8 & 17.8 & 17.8 & 17.8 \\
\hline $\begin{array}{l}\mathrm{b}\left(\mathrm{s} / \mathrm{mm}^{2}\right) \\
1-\mathrm{e}^{-\mathrm{bD}} \text { (brain) }\end{array}$ & 1.27 & 0.34 & 0.085 & 0.021 & 0.005 \\
\hline $\begin{array}{c}(\%) \\
1-\mathrm{e}^{-\mathrm{bD}} \text { (CSF) }\end{array}$ & 0.13 & 0.034 & 0.009 & 0.002 & $5 e-4$ \\
\hline (\%) & 0.32 & 0.085 & 0.021 & 0.005 & 0.001 \\
\hline
\end{tabular}


Table 2

Parameters that Describe the Quantity of Tagged Magnetization

\begin{tabular}{|c|c|c|c|}
\hline Method & $\tau(\mathrm{ms})$ & $M_{Z B}$ & $\tau \mathrm{M}_{\mathrm{ZB}}(\mathrm{ms})$ \\
\hline VSASL & $\mathrm{TI}=1600 \mathrm{~ms}$ & $e^{-T E_{\text {tag }} / T_{2}}\left(1-e^{-T R / T_{1}}\right)=0.77$ & 1230 \\
\hline VSASL + BGS & $\mathrm{TI}=1600 \mathrm{~ms}$ & $\begin{array}{l}e^{-T E_{\text {tag }} / T_{2}}\left(1-2 e^{-A / T_{1}}+2 e^{-B / T_{1}}-e^{-\left(T R-T I / T_{1}\right.}\right)=0.48 \\
A=T R-T I+T I_{B G S 2} B=T R-T I+T I_{B G S 1}\end{array}$ & 770 \\
\hline PQ2 & $\mathrm{TI}_{1}=700 \mathrm{~ms}$ & 2 & 1400 \\
\hline
\end{tabular}

\section{Methods for Restricting the Image to $V<V_{c}$}

Two methods have been proposed for restricting the spins that appear in the image acquisition to $V<V_{c}$. One method is to use VS gradients in the image acquisition sequence itself (11), which can be implemented as a pair of flow weighting gradients applied after the excitation pulse and prior to data acquisition. We refer to this as a VS acquisition (VSA). Another method is to use a second VS pulse train, applied at time TI, identical to that which is used for tagging, to saturate the spins with $V V_{C}$ at time TI in both tag and control images $(11,13)$. Because this second method is analogous to the method by which QUIPSS II (5) controls the temporal width of the tagged bolus in PASL, we refer to this method as VSASL/QUIPSS II. An optional additional delay can be inserted before image acquisition to allow for tagged blood to flow further down the vascular tree. A delay of $100 \mathrm{~ms}$ was added in the example shown below.

\section{Dependence on the Direction of Velocity Encoding}

The VSASL methods described above employ VS tagging along one direction at a time, and thus are sensitive only to the component of blood flow that is along the tagging direction. Because of the tortuosity of the vascular tree, any component of velocity can increase, decrease, or change sign as the spins flow toward target tissues. To investigate the effects of changes in the direction of velocity encoding, we performed VSASL with encoding along the three principal directions and for a range of values of $V_{c}$ from 1 to $16 \mathrm{~cm} / \mathrm{s}$.

\section{Perfusion Quantitation}

For PASL methods, the signal difference $\Delta S$ between tag and control images can be expressed approximately as:

$$
\Delta \mathrm{S}=\mathrm{S}_{O B} \mathrm{M}_{Z B} \tau \cdot \mathrm{CBFe} e^{-T I / T_{1}} e^{-T E / T_{2}}
$$

where $S_{O B}$ is the MR signal of a voxel full of fully relaxed arterial blood, $M_{Z B}$ is the difference in longitudinal magnetization of arterial blood between tag and control states immediately after the tag/control pulse (normalized to fully relaxed blood), $\tau$ is the temporal width of the tagged bolus, and the exponential terms describe the $T_{1}$ relaxation of the tag during TI and the $T_{2}$ relaxation during TE (26). Clearance of the tag by venous outflow and water exchange are neglected here. $S_{O B}$ is difficult to measure directly, but we estimate $S_{O B}$ by using the MR signal of CSF as a reference, and correcting for differences in $T_{2}$ and proton density between CSF and arterial blood (27). $M_{Z B}$ is dependent on the history of pulses that are experienced by the arterial blood, as well as the tagging method and the tagging efficiency $\alpha$. Unlike conventional ASL, in which the tagging and imaging regions are spatially separated, VSASL allows for tagging of blood that is already in the imaging region, and the imaging pulses themselves can therefore affect the arterial spin history. For spins that were saturated by imaging pulses in the previous TR period, $M_{Z B}=\alpha\left(1-e^{-\left(T R-T T / T_{1}\right.}\right)$ neglecting the time for image acquisition, while spins that were outside of the imaging area at the time of the previous image acquisition will have $M_{Z B}=\alpha\left(1-e^{-T R / T_{1}}\right)$ in the absence of BGS. In general, the amount of blood entering a voxel from inside relative to that entering from outside the imaging area is not known. For 2D imaging over a limited number of axial slices in healthy subjects, the approximation that all tagged blood is entering from outside the imaging area is a good one. However, for large imaging regions or pathology in which slow flow conditions may exist, a large fraction of tagged blood may be tagged within the imaging region, and to quantify CBF it is necessary to minimize or eliminate this source of uncertainty. This can be accomplished by using long TR, so that all spins are nearly fully relaxed after imaging and prior to the next tag or control pulse, or using a global saturation pulse immediately after image acquisition to reset the magnetization of all spins. With BGS, which we have implemented using spatially nonselective inversion pulses, the magnetization of all spins are near zero at the time of image acquisition, and incidentally serves a similar function to a nonselective post saturation pulse. For the ASL methods used here, expressions for $\tau$ and $M_{Z B}$ are shown in Table 2, along with numerical values for the imaging parameters used in the current experiments. Perfect inversion pulses were assumed for BGS, and because this was a 2D acquisition with only three slices in a normal subject, in the case of VSASL without BGS, all inflowing blood was assumed to enter the imaging region after the previous image acquisition.

\section{Imaging Parameters}

\section{General}

Five heathy subjects (two males and three females, 23-42 years old) were scanned after they provided informed consent according to a protocol approved by our institutional review board. All imaging was performed on a General Electric 3 Tesla whole-body imaging system using a commercial eight-channel head coil and the following imaging parameters: single-shot 2D spin-echo spiral acquisition; $\mathrm{TE}=16 \mathrm{~ms} ; \mathrm{TR}=3000 \mathrm{~ms} ; \mathrm{TI}=1600 \mathrm{~ms} ; 64 \times 64$ regridded matrix; FOV $=24 \mathrm{~cm} ; 3-8-\mathrm{mm}$ slices with $8-\mathrm{mm}$ gap; and fat presaturation. 
Table 3

Measured Gray Matter CBF*

\begin{tabular}{|c|c|c|c|c|}
\hline Subject & PICORE/QUIPSS II & PICORE/QUIPSS II + BGS & VSASL & VSASL + BGS \\
\hline 1 & $84.3(35.7)$ & $63.9(19.1)$ & $71.6(57.6)$ & $84.7(38.5)$ \\
\hline 2 & $75.4(29.5)$ & $67.0(15.2)$ & $52.3(36.7)$ & $69.3(27.4)$ \\
\hline 3 & 77.6 (45.2) & 50.0 (19.3) & $71.0(66.1)$ & $66.3(34.6)$ \\
\hline 4 & $75.0(40.2)$ & $54.2(14.6)$ & $46.8(50.8)$ & $59.3(27.7)$ \\
\hline 5 & $69.1(30.0)$ & 57.8 (15.2) & $42.8(48.5)$ & $59.9(27.2)$ \\
\hline Average & $76.3(36.1)$ & $58.6(16.7)$ & $56.9(51.9)$ & $67.9(31.1)$ \\
\hline
\end{tabular}

*Values are CBF in $\mathrm{ml} / 100 \mathrm{ml} / \mathrm{min}$ (temporal SD).

\section{VSASL-PICORE/QUIPPSS II Comparison}

Five subjects were scanned with both the VSASL and PICORE/QUIPSS II (PQ2) ASL methods, each with and without BGS, for a quantitative comparison of VSASL with an established PASL method. A total of 100 images were acquired using each method, alternating between tag and control states, for a scan time of $300 \mathrm{~s}$, and the order of scans was randomized across subjects. Other parameters were as follows:

VSASL: $V_{c}=2 \mathrm{~cm} / \mathrm{s}$ with flow encoding along the superior-inferior direction. $\mathrm{TE}_{\text {tag }}$ was $20 \mathrm{~ms}$. When BGS was used, nonselective adiabatic inversion pulses were applied $1550 \mathrm{~ms}$ and $450 \mathrm{~ms}$ prior to image acquisition.

PQ2: $\mathrm{TI}_{1}=700 \mathrm{~ms}$. When BGS was used, slab-selective adiabatic inversion pulses covering the imaging and tagging regions were applied $1000 \mathrm{~ms}$ and $300 \mathrm{~ms}$ prior to image acquisition.

\section{Calculation of $C B F$}

CBF was calculated using Eq. [1] and the values of $\tau$ and $M_{z b}$ from Table 2 . The $T_{1}$ and $T_{2}$ of arterial blood at $3 \mathrm{~T}$ were assumed to be $1664 \mathrm{~ms}$ (28) and $275 \mathrm{~ms}$ (29), respectively.

\section{GM ROI}

We obtained a GM ROI for each subject by calculating quantitative CBF maps for each of the four ASL methods, averaging the maps across the four methods, and applying to the averaged map a threshold for voxels with $\mathrm{CBF}>40$ $\mathrm{ml} / 100 \mathrm{ml} / \mathrm{min}$. This ROI was then used to calculate the values shown in Table 3. While this ROI creates a bias toward high values for $\mathrm{CBF}$, it selects voxels that are representative of GM in normal subjects, and does not create a strong bias toward any of the ASL methods.

\section{ASL Signal Stability}

To construct a time series of CBF maps, each image was subtracted from the average of the preceding and following images, and scaled using Eq. [1] to form a CBF time series (30). The stability of this time series was characterized by the standard deviation (SD) through time of each voxel, expressed in absolute CBF units.

To demonstrate the effects of variations in $V_{C}$, direction of velocity encoding, BGS, VSA, and VSASL/QUIPSS II, one subject was scanned with a wide range of these parameters in a single scan session. For each scan 120 images were acquired, the first 40 of which were velocity-encoded in the left-right (X) direction, the next 40 in the anteriorposterior (Y) direction, and the last 40 in the superiorinferior (Z) direction. PQ2 scans of 120 images were acquired for comparison.

\section{RESULTS}

Quantitative CBF maps and temporal SD maps calculated from VSASL and PQ2 data are shown for five subjects in Fig. 3. The differences in temporal SD outside the head are due to scalings that are required to present these data in CBF units (see Table 2). For a GM ROI the mean CBF and temporal SD of CBF are tabulated in Table 3. These data are averaged across all three slices (for brevity, only the lowest slice is shown in Fig. 3).

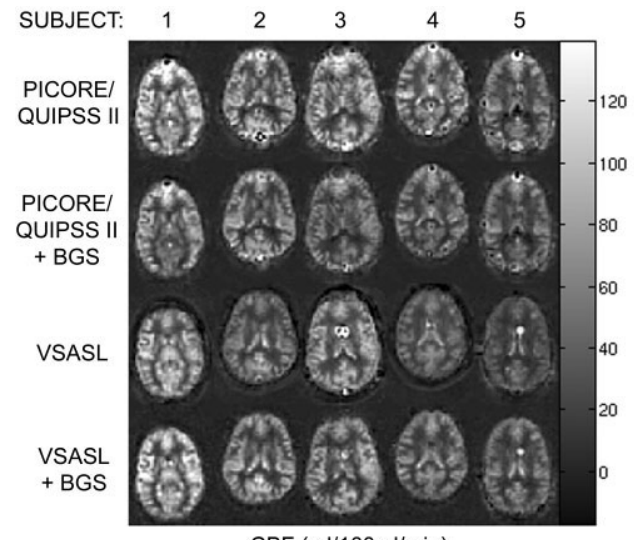

$\mathrm{CBF}(\mathrm{ml} / 100 \mathrm{ml} / \mathrm{min})$

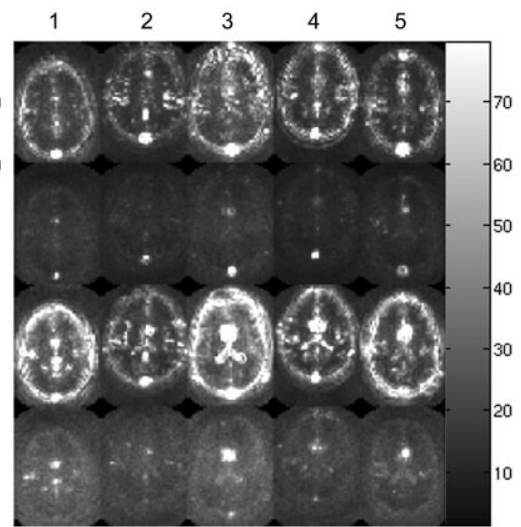

TEMPORAL SD of CBF signal ( $\mathrm{ml} / 100 \mathrm{ml} / \mathrm{min})$
FIG. 3. Comparison of VSASL with PICORE/QUIPSS II across five subjects. The left panel shows calculated CBF averaged across 100 images (50 tag/control pairs). The right panel shows the temporal SD of the time series of calculated CBF images. The mean values from these images over a GM ROI are tabulated in Table 3. 
FIG. 4. From left: VSASL with velocity encoding in the $L / R(X), A / P(Y)$, and $S / I(Z)$ directions; mean of $X-,, Y-$, and Z-encoding; SD across directions; SD across time series (noise); and mean/noise (SNR). All images in the first four columns are on the same absolute scale. From top: VSASL BGS, and $V_{c}=[1$ $\left.\begin{array}{lllll}2 & 4 & 8 & 16\end{array}\right] \mathrm{cm} / \mathrm{s}$, VSASL without BGS, VSASL with QUIPSS II style velocity selection prior to image, PASL using PQ2, and with and without BGS. Each of the images in the first three columns were acquired in $120 \mathrm{~s}$, and the PQ2 images were acquired in $360 \mathrm{~s}$.

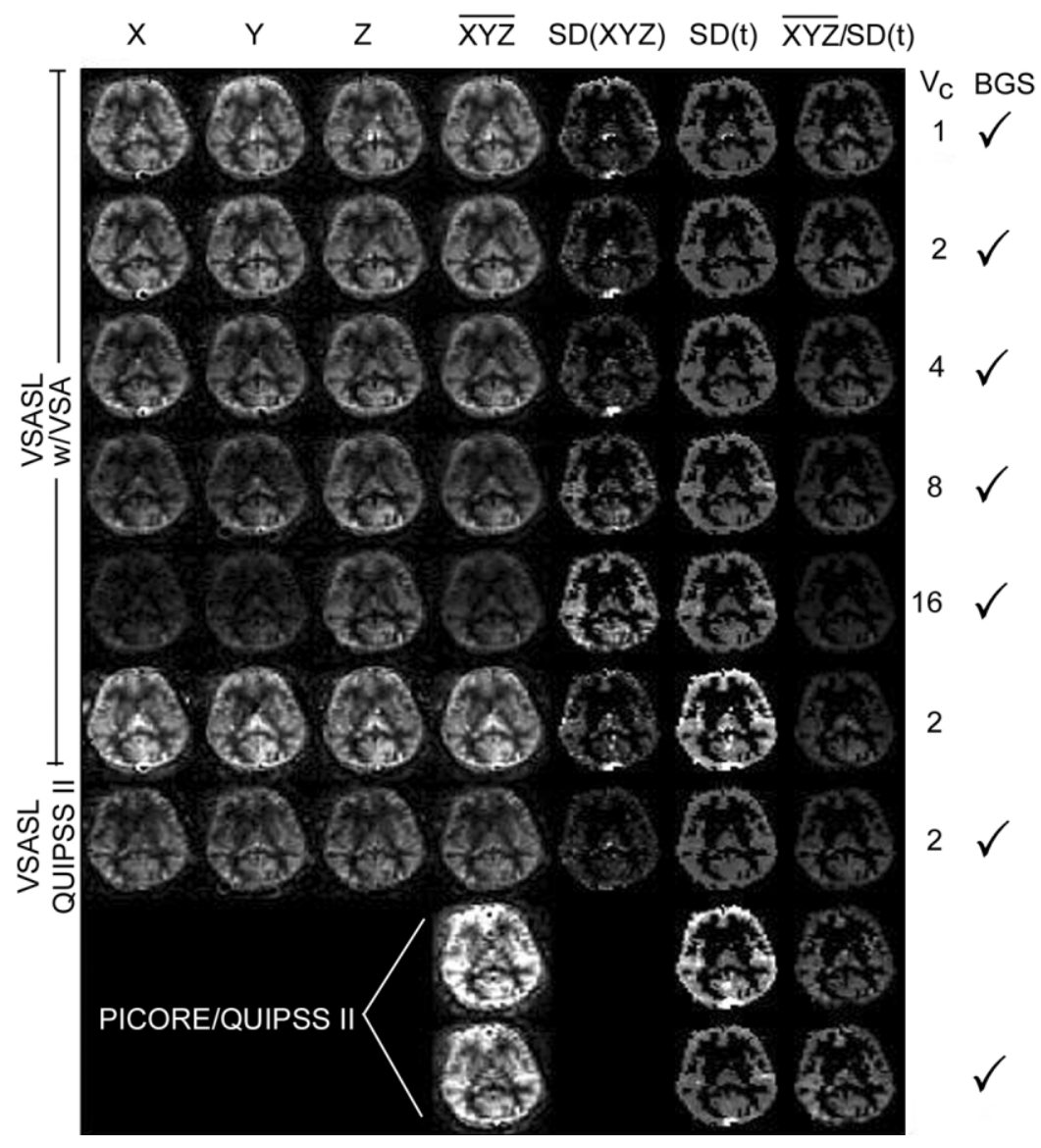

Examples of the dependence of the VSASL signal on the encoding direction, $V_{c}$, BGS, and VSA vs. VSASL/QUIPSS II are shown in Fig. 4. In this figure, data in the first four columns are presented as raw signal intensity after tag/ control subtraction, rather than calculated CBF units, and are all on the same scale. The rightmost column, labeled BGS, denotes which scans were acquired using BGS. The mean signal, SD, and the ratio of the two are shown in the fourth, sixth, and seventh columns, respectively, and visually demonstrate some the findings summarized in Table 3. PQ2 data are shown in the bottom two rows for comparison. The second and sixth rows differ in the use of BGS but are otherwise the same. Note in these rows that while the raw signal (XYZ) is higher without BGS, the SNR (characterized by XYZ/SD(t)) is higher with BGS. The column labeled SD(XYZ) is the SD across encoding directions, and is a measure of anisotropy with respect to encoding directions. Note that the VSASL data become markedly anisotropic for larger values of $V_{C}$. This is apparent in the raw images, where the VSASL signal for $\mathrm{X}$ and $\mathrm{Y}$ encoding becomes progressively reduced at higher $V_{c}$, and also in the higher values of $\mathrm{SD}$ (XYZ), which increases with $V_{c}$.

\section{DISCUSSION}

For the four ASL methods shown in Fig. 3 and Table 3, the trends in both $\mathrm{CBF}$ and temporal SD of CBF were relatively consistent across subjects. In the absence of BGS, the cal- culated CBF for VSASL was 25(8-38)\% lower than that for $\mathrm{PQ} 2$, while in the presence of BGS, the calculated CBF for VSASL was $16(3-32) \%$ higher than that for PQ2. This discrepancy may be due to systematically low CBF values for VSASL without BGS, although the cause of this is not well understood. Gross artifacts in the CBF maps in the form of negative values outside the brain are apparent only in the VSASL images without BGS, and may be related to a negative bias in those images. Also, the SNR, expressed as the mean CBF divided by the temporal SD, was a factor of 2 lower for VSASL without BGS (1.1) than for any of the other techniques. The SNR for VSASL with BGS (2.2) is similar to that of PQ2 without BGS (2.1), but lower than that of PQ2 with BGS (3.5). The mean CBF for VSASL with BGS was intermediate between the PQ2 values with and without BGS. For PQ2, the reduction in apparent CBF with BGS was $23 \%$, and is roughly consistent with previous measurements of the efficiency of BGS in ASL as summarized in Ref. 23. For VSASL, the use of BGS increased the calculated CBF by $19 \%$. However, unlike PQ2, the BGS pulses in VSASL are necessarily nonselective, and therefore affect the signal equations and the CBF calculation. Thus, the effect of BGS in VSASL is not a simple scaling by the inversion efficiency; rather, it is a more complicated effect of the BGS pulses on the spin history of arterial blood.

Two additional artifacts are of note. First, CSF flow artifacts are apparent in the VSASL CBF maps for subjects 3 and 5 in the ventricles, and are reduced with BGS. 
Second, several focal artifacts, both positive and negative, appear around the perimeter of the PQ2 CBF maps for all subjects, but are not apparent in the VSASL images. These are presumably artifacts from intravascular signal.

VSASL is currently the only ASL method that has the potential to provide quantitative measures of CBF in the presence of slow or collateral flow. Because the arterial tagging is spatially nonselective, anatomical coverage with VSASL is not limited by arterial transit parameters. The method is therefore amenable to $3 \mathrm{D}$ or rapid multislice image acquisition methods, and can provide whole-brain coverage with uniform sensitivity to perfusion. For these reasons, VSASL is of particular interest for clinical applications, such as stroke.

A critical parameter in the design of a VSASL pulse sequence is the choice of the cutoff velocity $V_{c}$. This is the velocity above which arterial blood will be tagged, and below which spins will be included in the VSASL image. Thus the VSASL signal consists of tagged magnetization that stretches from arteries whose flow velocity is just below $V_{c}$, downstream into the target tissues. If $V_{C}$ is high, then arterial spins with velocities just below $V_{C}$ will be upstream in the arterial tree, producing intravascular signal in larger arteries that does not reflect local perfusion. If $V_{c}$ is too low, several other sources of artifacts will appear. These include sensitivity to subject motion and physiological pulsations, diffusion sensitivity, and sensitivity to CSF flow, as described above. The optimum $V_{C}$ is one that is just low enough that the VSASL signal is localized well enough to the target tissue to answer the question at hand. Further reduction of $V_{c}$ increases image artifacts and systematic errors without benefit. In the normal brain, the 30-50 $\mu \mathrm{m}$ arterioles that penetrate the cortical surface have flow velocities in the $1-2 \mathrm{~cm} / \mathrm{s}$ range (31). This provides a practical target for $V_{C}$ in the brain and ensures that the VSASL signal will appear in or on the surface of the GM, and not in more-distant upstream arteries. In pathology or in other tissues, the relevant flow velocities in areas of interest may be higher or lower, and an estimate of the range of velocities present may be an important part of the optimization of VSASL techniques for stroke and other pathologies.

For VSASL, a key feature of the vasculature is that, in general, arterial blood decelerates, while venous blood accelerates. This is important for two reasons: first, it allows for the use of a velocity cutoff as a threshold through which one can quantify the passage of blood, and thus allows for spatially nonselective tagging; second, it provides an automatic means of excluding venous blood from the VSASL signal. There are clearly examples of locations in the brain at which the component of arterial blood flow velocity in any given direction is not monotonically decreasing, such as through stenoses or along curving arteries. However, it is possible to use a cutoff velocity $V_{c}$ that is low enough so that the method will be insensitive to the geometry of the larger arteries. Empirically, the VSASL signal appears to be insensitive to the direction of velocity encoding for values of $V_{c}$ below approximately $4 \mathrm{~cm} / \mathrm{s}$ (see Fig. 4).

The double sech VS tagging scheme described here differs from the original hard pulse scheme $(13,18)$ primarily in that it provides improved robustness to $B_{1}$ inhomoge- neity, and the velocity selectivity profile is nominally unchanged. Other VS pulse trains have been explored. Norris and Schwartzbauer (17) described the use of pulse trains of the form $\left(a_{i}-G_{i}-180-G_{i}-\left(-a_{i}\right)\right)_{\mathrm{n}}$ to provide velocity selectivity with tunable velocity profiles, and we have modified this method using a hyperecho pulse train (32) to achieve the same functionality with shorter pulse trains (12). While these methods can in principle produce improved velocity selectivity, they are exquisitely sensitive to variations in flip angles, and even with the use of BIR-4 rotation pulses (33) to provide $B_{1}$ insensitivity, we have not found the robustness of these pulse trains to be adequate for VSASL. However, with more robust plane rotation pulses, these pulse trains may be of interest in the future. Of particular interest is the potential for these pulse trains to create a very flat velocity response in the $0-5 \mathrm{~mm} / \mathrm{s}$ velocity range, where most subject motion is likely to occur. A Fourierbased method for generating VS pulse trains with arbitrary velocity selectivity was recently introduced (34), but for gradient strengths that are available in human imaging systems this method is not currently useful for generating velocity selectivity for velocities low enough for VSASL. However, this method may be of interest for small-animal systems, where much higher gradient performance is available, and the effect of tuned VS pulses in VSASL can be tested in animal models.

Areas of development in VSASL that remain to be addressed include the implementation of efficient 3D methods for image acquisition, and testing of the method in pathology. Our laboratory is currently conducting studies in these areas. In addition, it is possible that under pathological conditions the optimum value of $V_{c}$, as well as the dependence on the direction of encoding, will be different than in normal subjects, and that the method will require retuning for different applications.

\section{ACKNOWLEDGMENTS}

The authors thank Karam Sidaros and Elizabeth Yoder for helpful input in the development of VSASL.

\section{REFERENCES}

1. Edelman RR, Chen Q. EPISTAR MRI: multislice mapping of cerebral blood flow. Magn Reson Med 1998;40:800-805.

2. Kim S-G, Tsekos NV. Perfusion imaging by a flow-sensitive alternating inversion recovery (FAIR) technique: application to functional brain imaging. Magn Reson Med 1997;37:425-435.

3. Kwong KK, Chesler DA, Weisskoff RM, Donahue KM, Davis TL, Ostergaard L, Campbell TA, Rosen BR. MR perfusion studies with T1weighted echo planar imaging. Magn Reson Med 1995;34:878-887.

4. Wong EC, Buxton RB, Frank LR. Implementation of quantitative perfusion imaging techniques for functional brain mapping using pulsed arterial spin labeling. NMR Biomed 1997;10:237-249.

5. Wong EC, Buxton RB, Frank LR. Quantitative imaging of perfusion using a single subtraction (QUIPSS and QUIPSS II). Magn Reson Med 1998;39:702-708.

6. Alsop DC, Detre JA. Reduced transit-time sensitivity in noninvasive magnetic resonance imaging of human cerebral blood flow. J Cereb Blood Flow Metab 1996;16:1236-1249.

7. Alsop DC, Detre JA. Multisection cerebral blood flow MR imaging with continuous arterial spin labeling. Radiology 1998;208:410-416.

8. Detre JA, Zhang W, Roberts DA, Silva AC, Williams DS, Grandis DJ, Koretsky AP, Leigh JS. Tissue-specific perfusion imaging using arterial spin labeling. NMR Biomed 1994;7:75-82. 
9. Ye FQ, Mattay VS, Jezzard P, Frank JA, Weinberger DR, McLaughlin AC. Correction for vascular artifcats in cerebral blood flow values measured by using arterial spin tagging techniques. Magn Reson Med 1997;37:226-235.

10. Neumann-Haefelin T, Wittsack HJ, Wenserski F, Siebler M, Seitz RJ, Modder U, Freund HJ. Diffusion- and perfusion-weighted MRI. The DWI/PWI mismatch region in acute stroke. Stroke 1999;30:1591-1597.

11. Wong EC, Liu TT, Sidaros K, Frank LR, Buxton RB. Velocity selective arterial spin labeling. In: Proceedings of the 10th Annual Meeting of ISMRM, Honolulu, HI, USA, 2002. p 621.

12. Wong EC, Cronin M. Velocity selective arterial spin labeling using an adiabatic hyperecho pulse train. In: Proceedings of the 11th Annual Meeting of ISMRM, Toronto, Canada, 2003. p 2181.

13. Duhamel G, de Bazelaire C, Alsop DC. Evaluation of systematic quantification errors in velocity-selective arterial spin labeling of the brain. Magn Reson Med 2003;50:145-153.

14. Wong EC. Time efficient CSF suppressed velocity selective ASL using a T2-FLAIR preparation. In: Proceedings of the 12th Annual Meeting of ISMRM, Kyoto, Japan, 2004. p 711.

15. Cronin MV, Wong EC, Wu W-C, Frank LR. Directional dependence in velocity selective arterial spin labeling. In: Proceedings of the Annual Meeting of ISMRM, Kyoto, Japan, 2004. p 719.

16. Wu W-C, Wong EC. Intravascular effects in velocity-selective arterial spin labeling: the choice of transit delay and cutoff velocity. In: Proceedings of the 13th Annual Meeting of ISMRM, Miami, FL, USA, 2005. p 1157.

17. Norris DG, Schwartzbauer C. Velocity selective radiofrequency pulse trains. J Magn Reson 1999;137:231-236.

18. Wong EC, Liu TT, Sidaros K, Frank LR, Buxton RB. Velocity selective arterial spin labeling. In: Proceedings of the 10th Annual Meeting of ISMRM, Honolulu, HI, USA, 2002. p 621.

19. Conolly S, Glover G, Nishimura D, Macovski A. A reduced power selective adiabatic spin-echo pulse sequence. Magn Reson Med 1991; 18:28-38.

20. Conolly S, Nishimura D, Macovski A, Glover G. Variable-rate selective excitation. J Magn Reson 1988;78:440-458.

21. King KF, Gai N, Ganin A, Glover G. Correction for gradient amplifier hysteresis artifacts in spiral scans. In: Proceedings of the 8th Annual Meeting of ISMRM, Denver, CO, USA, 2000. p 336.
22. Reese TG, Heid O, Weisskoff RM, Wedeen VJ. Reduction of eddycurrent-induced distortion in diffusion MRI using a twice-refocused spin echo. Magn Reson Med 2003;49:177-182.

23. Garcia DM, Duhamel G, Alsop DC. The efficiency of background suppression in arterial spin labeling. In: Proceedings of the 12th Annual Meeting of ISMRM, Kyoto, Japan, 2004. p 1360.

24. St Lawrence KS, Frank JA, Bandettini PA, Ye FQ. Noise reduction in multi-slice arterial spin tagging imaging. Magn Reson Med 2005;53: 735-738.

25. Ye FQ, Frank JA, Weinberger DR, McLaughlin AC. Noise reduction in 3D perfusion imaging by attenuating the static signal in arterial spin tagging (ASSIST). Magn Reson Med 2000;44:92-100.

26. Buxton RB, Frank LR, Wong EC, Siewert B, Warach S, Edelman RR. A general kinetic model for quantitative perfusion imaging with arterial spin labeling. Magn Reson Med 1998;40:383-396.

27. Floyd TF, Ratcliffe SJ, Wang J, Resch B, Detre JA. Precision of the CASL-perfusion MRI technique for the measurement of cerebral blood flow in whole brain and vascular territories. J Magn Reson Imaging 2003;18:649-655.

28. Lu H, Clingman C, Golay X, van Zijl PC. Determining the longitudinal relaxation time (T1) of blood at 3.0 Tesla. Magn Reson Med 2004;52: $679-682$

29. Stanisz GJ, Odrobina EE, Pun J, Escaravage M, Graham SJ, Bronskill MJ Henkelman RM. T1, T2 relaxation and magnetization transfer in tissue at 3T. Magn Reson Med 2005;54:507-512.

30. Liu TT, Wong EC. A signal processing model for arterial spin labeling functional MRI. Neuroimage 2005;24:207-215.

31. Kobari M, Gotoh F, Fukuuchi Y, Tanaka K, Suzuki N, Uematsu D. Blood flow velocity in the pial arteries of cats, with particular reference to the vessel diameter. J Cereb Blood Flow Metab 1984;4:110-114.

32. Hennig J, Scheffler K. Hyperechoes. Magn Reson Med 2001;46:6-12.

33. Garwood M, Ke Y. Symmetric pulses to induce arbitrary flip angles with compensation for RF inhomogeneity and resonance offsets. J Magn Reson 1991;94:511-525.

34. de Rochefort L, Maitre X, Bittoun J, Durand E. Velocity-selective RF pulses in MRI. Magn Reson Med 2006;55:171-176. 